\title{
Clinical impact and cost analysis of the use of either the Xpert MTB Rif test or sputum smear microscopy in the diagnosis of pulmonary tuberculosis in Rio de Janeiro, Brazil
}

\author{
Anália Zuleika de Castro ${ }^{[1]}$, Adriana Rezende Moreira ${ }^{[1],[2], ~ J a q u e l i n e ~ O l i v e i r a ~}{ }^{[2],}$ \\ Paulo Albuquerque Costa[2],[3], Carolyne Lalucha Alves Lima Da Graça ${ }^{[1]}$, \\ Mauricio de Andrade Pérez ${ }^{[4],[5]}$, Afrânio Kritski[ ${ }^{[1],[2]}$ and Maria Claudia Vater ${ }^{[4]}$
}

\begin{abstract}
[1]. Programa Pós-Graduação em Clínica Médica, Faculdade de Medicina, Universidade Federal do Rio de Janeiro, Rio de Janeiro, RJ, Brasil.
[2]. Programa Acadêmico de Tuberculose, Faculdade de Medicina e do Complexo Hospitalar, Instituto de Doenças do Tórax,

Hospital Universitário Clementino Fraga Filho, Universidade Federal do Rio de Janeiro, Rio de Janeiro, RJ, Brasil.

[3]. Policlínica Augusto Amaral Peixoto, Departamento de Saúde Municipal, Rio de Janeiro, RJ, Brasil.

[4]. Instituto de Estudos em Saúde Coletiva, Universidade Federal do Rio de Janeiro, Rio de Janeiro, RJ, Brasil.
\end{abstract}

[5]. Curso de Medicina, Faculdades Souza Marques, Rio de Janeiro, RJ, Brasil.

\begin{abstract}
Introduction: The molecular test Xpert MTB/RIF (Xpert) has been recommended for use in the diagnosis of pulmonary tuberculosis (PTB); however, data on the cost of incorporating it under routine conditions in high-burden countries are scarce. The clinical impact and costs incurred in adopting the Xpert test in routine PTB diagnosis was evaluated in a prospective study conducted from November 2012 to November of 2013, in the City of Rio de Janeiro, Brazil. Methods: The diagnostic and therapeutic cascade for TB treatment was evaluated using Xpert in the first stage (S1), and sputum smear microscopy (SSM) in the second stage (S2). The mean costs associated with each diagnostic test were calculated including equipment, human resources, supplies, and infrastructure. Results: We included 232 subjects with probable TB $(\mathrm{S} 1=87 ; \mathrm{S} 2=145)$. The sensitivities of Xpert and SSM were $91.7 \%(22 / 24)$ and $79.1 \%$ (34/43), respectively. The median time between triage and TB treatment initiation in S1 $(\mathrm{n}=24)$ was 14.5 days (IQR 8-28.0) and in $\mathrm{S} 2(\mathrm{n}=43)$ it was 8 days [interquartile range (IQR) 6-12.0]. The estimated mean costs per examination in S1 and S2 were US\$24.61 and US\$6.98, respectively. Conclusions: Compared with SSM, Xpert test showed a greater sensitivity, but it also had a time delay with respect to treatment initiation and a higher mean cost per examination.
\end{abstract}

Keywords: Diagnostic methods. Pulmonary tuberculosis. Cost analysis. Xpert MTB/RIFtest. Sputum smear microscopy.

\section{INTRODUCTION}

Tuberculosis (TB) has become one of the top 10 causes of death worldwide, being the major cause of mortality among infectious diseases in $2015^{1,2}$, with about 10.4 million people becoming infected with TB, and 1.8 million dying from this disease [including 0.4 million patients with human immunodeficiency virus (HIV)]. Since 2008, the World Health Organization (WHO) has prioritized the early diagnosis of TB through new molecular technologies, which allow for the adoption of appropriate measures, for both treatment and infection control in the community ${ }^{3,4}$.

Corresponding author: Dr. Afrânio Kritski.

e-mail:kritskia@gmail.com

Received 3 March 2018

Accepted 30 July 2018
Since 2010,the WHO has recommended the Xpert MTB/RIF test (Xpert) as a substitute for the sputum smear microscopy (SSM) test for TB diagnosis in adults and children ${ }^{1}$.In clinical trials that analyzed the use of Xpert in TB diagnosis in comparison with SSM, the Xpert test increased the number of bacteriologically-confirmed TB patients, reduced the time between triage and treatment initiation, but did not reduce either mortality or the number of patients lost to follow-up 5 .In addition, a recent survey in South Africa, using primary data obtained under routine conditions, found that the use of Xpert was not cost-effective ${ }^{6}$. These results indicate the need for studies that can evaluate the clinical impact and costs of incorporating new diagnostic technologies for $\mathrm{TB}$, under routine conditions, in high-burden countries ${ }^{7-9}$.

In Brazil, owing to the low access to TB diagnosis in some regions ${ }^{10,11}$, higher rates of mortality per 100,000 inhabitants due to TB occur in the States of Rio de Janeiro, Pernambuco, and Amazonas, being, 5.0, 4.5, and 3.2 respectively ${ }^{1}$. 
In a clinical trial performed in the Cities of Rio de Janeiro and Manaus, using secondary data obtained from the Integrated Laboratory System (ILS) and Disease Notification system (DNS), compared to SSM, the use of Xpert resulted in a) a higher proportion of bacteriologically-confirmed TB, a shorter time to treatment initiation ${ }^{12}$, and a lower $\cos ^{13}$, but there was no impact on TB treatment outcomes ${ }^{14}$. Under this scenario, the Xpert test was introduced into the Brazilian Health System in $2013^{15}$.

In the present study, using primary data obtained from a health unit in Rio de Janeiro City, we compared the care process indicators related to the diagnostic and treatment cascades for patients with probable TB, and the mean costs incurred using either Xpert or SSM.

\section{METHODS}

Patients: A prospective, descriptive, analytical study was conducted from November 2012 to November 2013, at the Policlínica Augusto Amaral Peixoto (PAAP), a health unit located in Guadalupe neighborhood at Programmatic Area AP 3.3, in the city of Rio de Janeiro. Following the recommendations of the Ministry of Health, eligible patients were defined as having signs and/or symptoms suggestive of pulmonary TB, were older than 12 years of age, were either male or female, had initiated or not TB treatment at PAAP, and were residents of Rio de Janeiro City ${ }^{16}$. Subjects with a history of coughing, hemoptysis, and/ or abnormalities on a chest X-ray compatible with pulmonary $\mathrm{TB}$, who signed the informed consent form, and those that had their respiratory samples analyzed by Xpert or SSM were included. Subjects whose clinical samples were not submitted for mycobacterial culture, whose diagnosis was extra-pulmonary TB, or who had already started an anti-TB treatment were excluded.

Confirmed bacteriological TB was defined as a positive culture result, and Empirical TB treatment was defined when anti-TB treatment was started by a physician based on the patient's symptoms and clinical history, before having access to the laboratory test result ${ }^{16}$.

Ziehl-Neelsen staining and culture for mycobacteria were used, following standard laboratory recommendations and the Xpert test was performed according to the manufacturer's recommendations ${ }^{17,18}$. Cultures for mycobacteria were carried out in Lowenstein-Jensen or BACTEC ${ }^{\mathrm{TM}}$ MGIT $^{\mathrm{TM}} 960$ systems at the Mycobacteriology Laboratory of the University Hospital Clementino Fraga Filho [Hospital Univeristário Clementino Fraga Filho (HUCFF)], Federal University of Rio de Janeiro [Universidade Federal do Rio de Janeiro (UFRJ)], and the Evandro Chagas Clinical Research Institute [Instituto de Pesquisa Clinica Evandro Chagas, (IPEC)], Oswaldo Cruz Foundation [Fundação Oswaldo Cruz (FIOCRUZ)].The clinical samples were collected in two stages, stage 1 (Nov 2012-April 2013) and stage 2 (May-November 2013). In stage 1, Xpert was performed at the Souza Marques Family Clinic located in another neighborhood (Madureira), and in stage 2, owing to a lack of Xpert supplies, $\mathrm{SSM}$ was reintroduced for TB diagnosis at PAAP.

Anti-HIV testing was proposed for patients who started antiTB treatment, following the guidance of the Ministry of Health Standards Manual ${ }^{18}$. Lost to follow-up cases were called when the patient did not attend the health unit after two pre-scheduled, unfilled consultations. In cases of death, the medical records were evaluated to confirm its relationship to TB.

\section{Cost analysis}

Following the recommendation of the Ministry of Health for Health Technologies Evaluation ${ }^{19}$, the costs associated with Xpert and SSM were evaluated. The items evaluated were classified into four different categories: equipment, human resources, inputs, and infrastructure (electricity, safety, water, cleaning). The appropriation method was used to estimate the mean cost which was calculated by adding up all the monthly expenses of the Laboratory associated with the exam, divided by the number of exams carried out in one month. This had the advantages that it simplified calculations and the information was easy to obtain.

It should be noted that the calculation of the item infrastructure cost involved the use of the so-called apportionment method ${ }^{20}$. For this, the values of the unit's total assets were known, and the proportion used by each item in the laboratory was calculated. The item human resources, was calculated as follows: (salary of the professionals $\mathrm{X}$ amount of human resource per category $\mathrm{X}$ the average dedication devoted to the accomplishment of the procedure by category) / the total examinations. With regard to estimated inputs costs we used a series of parameters to compute the expenditure published elsewhere ${ }^{21}$, which inform the model, the average expenditure for each one to perform the exam. The expenditure of the input by exam was multiplied by its market price (at the time), divided by the total of exams made. In the case of equipment, the amount of equipment was multiplied by the price of the equipment used, discounting a depreciation rate of $5 \%$, obtained from its useful life divided by the total of examinations carried out in the month. These values were converted for US\$, according to Brazil's central bank exchange rate (November, 2013). ${ }^{22}$ The time (in days) from triage to TB treatment initiation was used as a measure of the effectiveness of the diagnostic procedure. A predicted cost effectiveness analysis that incorporates the loss, in terms of costs, and the gain, in terms of effectiveness, was performed using Xpert compared with the traditional technology, sputum smear microscopy.

\section{Statistical analysis}

We compared the socio-demographic data and the clinical characteristics between the patients in stage 1 and stage 2. For categorical data, percentages, chi square tests, and odds ratios were used. For variables, measures of central tendency, such as median and quartiles, were used. For analysis of the time between triage and process indicators linked to the diagnostic and therapeutic cascade, analyses were performed to compare continuous variables with normal and non-normal distributions, such as the t-test and Mann Whitney test. To verify if there was a difference between proportions, the $95 \%$ confidence interval was used. The analyses were performed using the statistical package SPSS, version 20.0.

\section{Ethical considerations}

The Research Ethics Committee of Faculdade de Medicina, Hospital Universitário Clementino Fraga Filho, Universidade Federal do Rio de Janeiro approved the study under number 019/07.17. 


\section{RESULTS}

During the study period, 411 subjects with probable TB were deemed eligible (146 in stage 1 and 265 in stage 2). Among these, $232(56.4 \%)$ were included in the study; 87 in stage 1 and 145 in stage 2 . TB treatment was initiated in $67(28.8 \%)$ patients; $24(27.6 \%)$ in stage 1 and $43(29.6 \%)$ in stage 2 . The Xpert test used in stage 1 was positive in 22/24 (91.7\%) patients, and the SSM used in stage 2 was positive in $34 / 43$ (79.1\%) patients (Figure 1).

From the data shown in Table 1, we observed that in both stages, there was a predominance of male patients $(70.2 \%)$, a black or brown skin color (77.1\%), economic class $\mathrm{C}$ and D (84.5\%), lower levels of schooling (87.5\%), and smokers or ex-smokers $(65.5 \%)$. In addition, there was a high rate of contact with pulmonary TB (34.9\%) and a past history of TB $(22.8 \%)$. The empirical treatment ratio was higher in stage 1 $(7.7 \%)$ than in stage $2(2.4 \%)$. No significant differences were observed when comparing stage 1 and 2 with respect to sociodemographic and clinical variables.

In stage 1, the time from the triage and laboratory results, receipt of the laboratory results by health professionals, and initiation of TB treatment were, 4, 13, and 14.5 days, respectively. In stage 2 , the time from the triage and laboratory results, receipt of the laboratory results by health professionals, and the initiation of TB treatment were, 3, 7, and 8 days, respectively. Comparing stage 1 to stage 2 , the times (in days) from triage and receipt of laboratory results by health professionals and treatment initiation were significantly longer with Xpert $(p<0.001)$, but there was no difference between triage and sputum collection and laboratory results release $(\mathrm{p}=0.06)$ (Table 2).

Table 3 shows that there was no difference between the TB treatment outcomes among the TB patients diagnosed in stage 1 or stage 2 . Among the 67 TB patients, $20(29.8 \%)$ were transferred to primary health units. Among the 47 patients who remained at PAAP, 8 patients were lost to follow-up (11.9\%).

The exams performed in stage 1 and 2 among the 232 patients were included in the cost analysis. In stage 1 and 2, 92 Xpert and 378 SSM exams were performed as a diagnostic routine, respectively. In stage 2, 213 SSMs were performed in the first exam and 152 in the second exam. On average, 31.3 tests per month were performed by Xpert and 70.2 tests by SSM. The estimated mean costs of Xpert and SSM were US\$24.61 and US\$ 6.98 per exam, respectively (Table 4). The highest cost categories associated with Xpert were supplies and equipment, whereas for SSM the highest cost category was human resources (Table 4).

A cost-effectiveness analysis was not performed, since the patients who attended in stage 1 (Xpert) took 14.5 days on average to start TB treatment, whereas the patients in stage 2 (SSM) took 8 days. In addition, the SSM has a lower mean cost relative to Xpert. That way, in this sample, a dominated analysis was characterized.

\section{DISCUSSION}

In our study, under routine conditions, patients who were analyzed using Xpert (stage 1) experienced an average of 14.5 days from the time triage to the initiation of TB treatment.

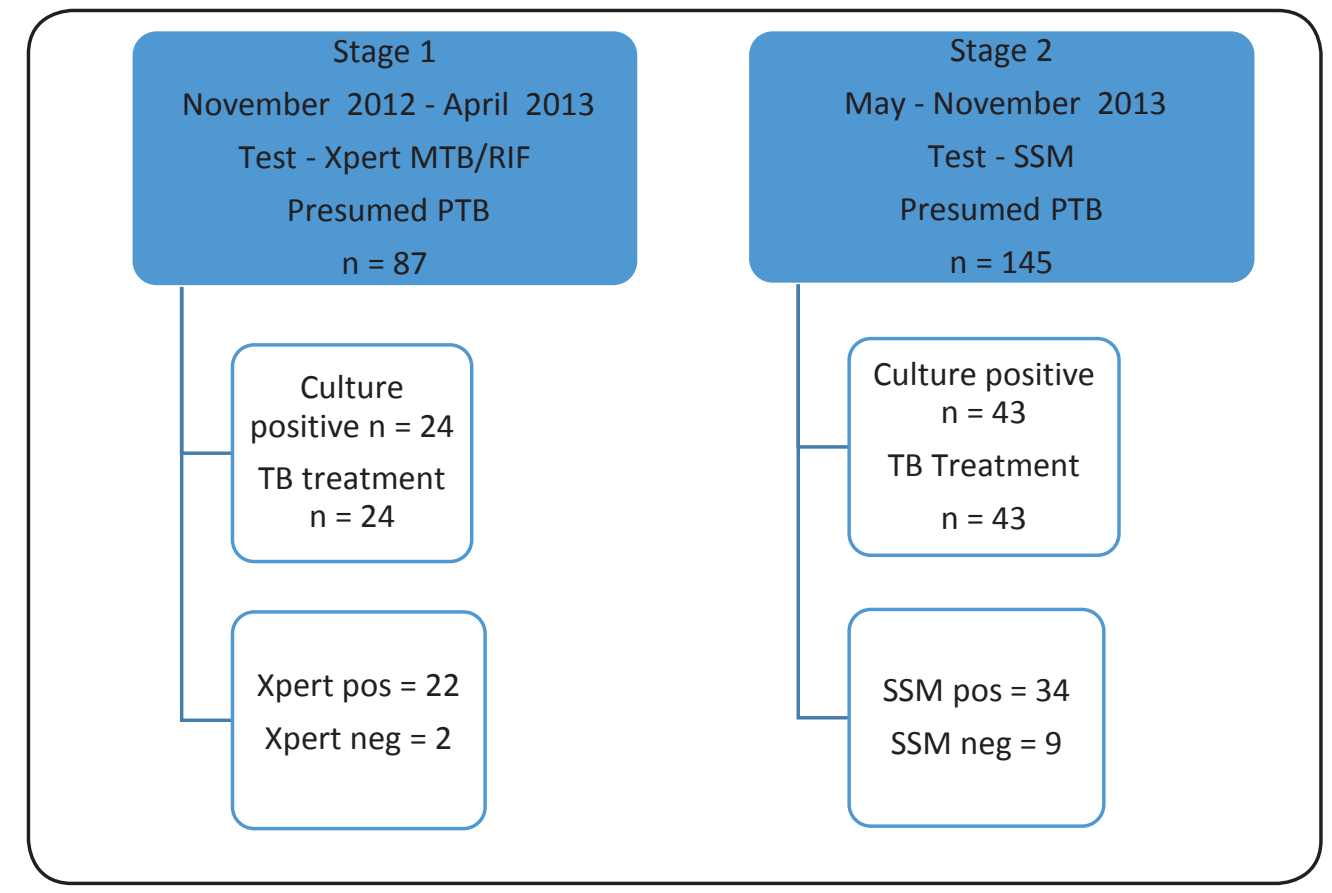

FIGURE 1: TB diagnosis and treatment cascade among presumed pulmonary tuberculosis cases during the study period using Xpert MTB RIF and sputum smear microscopy.Xpert MTB RIF: Xpert Mycobacterium Rifampicin; PTB:pulmonary tuberculosis; SSM: sputum smear microscopy; TB: tuberculosis. 
TABLE 1: Socio-demographic and clinical characteristics of patients with probable pulmonary tuberculosis treated at the health unit from November 2012 to November 2013.

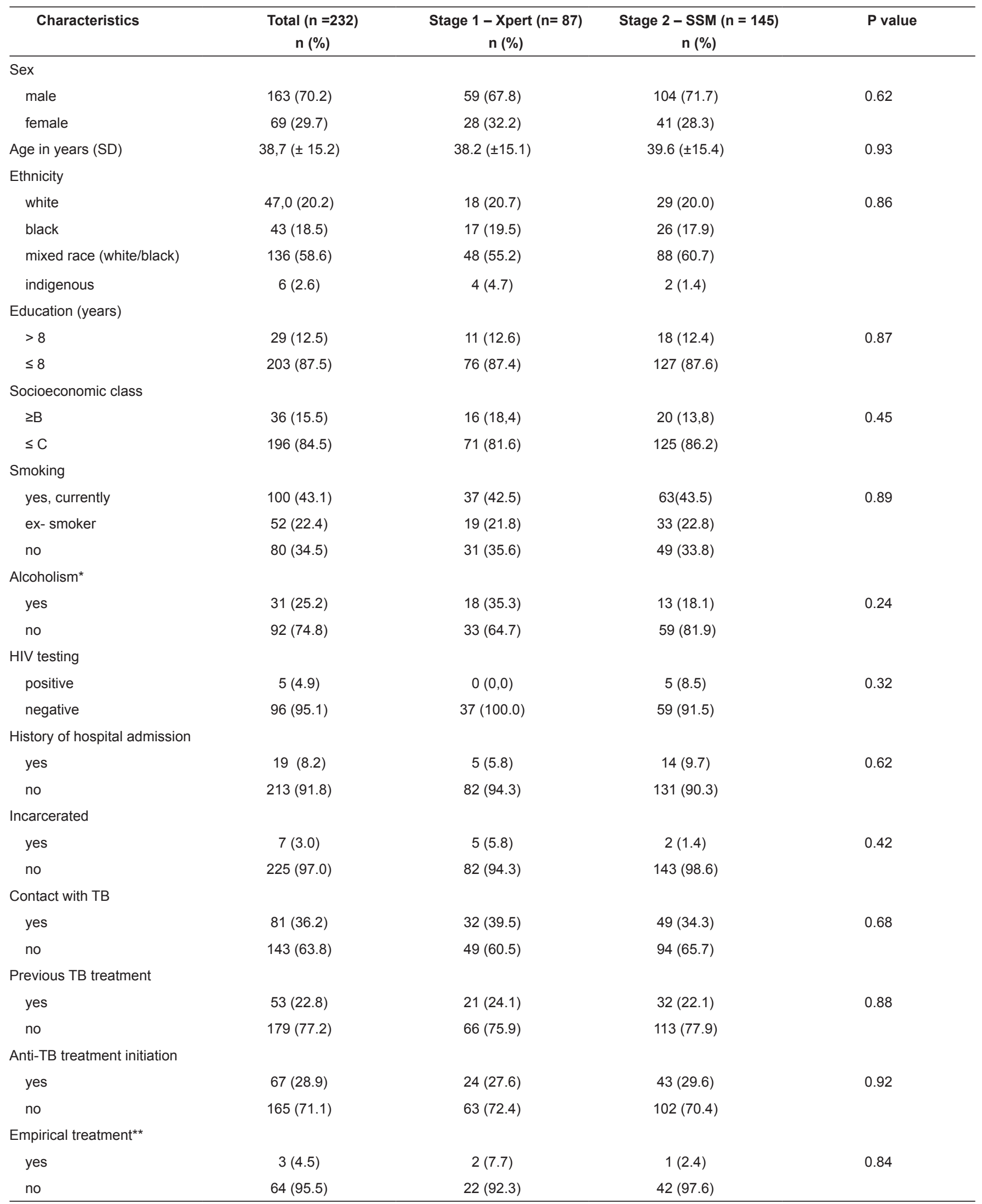

SSM: sputum smear microscopy. ${ }^{*}$ Empirical treatment was initiated before the laboratory test results; TB: tuberculosis. SD: standar deviation. ${ }^{* *}$ Alcoholism analyzed by the test CAGE (Cutting down, Annoyance by criticism, Guilty feeling, and Eye-openers). 
TABLE 2: Time (in days) between triage and other process indicators for TB diagnosis and treatment cascade among 232 patients with probable pulmonary TB, from November 2012 to November 2013.

\begin{tabular}{|c|c|c|c|c|c|c|}
\hline \multirow{2}{*}{ Process indicators } & \multicolumn{3}{|c|}{ Stage 1 (Xpert) } & \multicolumn{3}{|c|}{ Stage 2 (SSM) } \\
\hline & median (IQR) & median (IQR) & median (IQR) & median (IQR) & median (IQR) & median (IQR) \\
\hline \multirow[t]{2}{*}{ Sputum collection } & 0 & 0 & 0 & 0 & 0 & 0 \\
\hline & $(0-1)$ & $(0-1)$ & $(0-1)$ & $(0-0)$ & $(0-0)$ & $(0-0)$ \\
\hline & $(2-7)$ & $(2-8)$ & $(2-7)$ & $(2-5)$ & $(2-6)$ & $(2-5)$ \\
\hline \multirow{2}{*}{$\begin{array}{l}\text { Laboratory results } \\
\text { received by HCWs }\end{array}$} & 13 & 10.5 & 14 & 7 & 7 & $7(6-9)$ \\
\hline & $(8-22)$ & $(7-15)$ & $(8-24)$ & $(6-8)$ & $(6-8)$ & \\
\hline TB treatmentinitiation & & 14,5 & & & 8 & \\
\hline
\end{tabular}

TB: tuberculosis;SSM: sputum smear microscopy ;IQR: interquartile range. HCWs: health care workers.

TABLE 3: Treatment outcomes in patients diagnosed by Xpert in stage 1 and by SSM in stage 2, from November 2012 to November 2013.

\begin{tabular}{lcc}
\hline Effectiveness anti-TB treatment & Stage $\mathbf{1}(\mathbf{n = 2 4 )}$ & Stage 2 (n=43) \\
\hline Favorable (healing/complete treatment) & $12(75.0 \%)$ & $23(79.3 \%)$ \\
Unfavorable & $4(25.0 \%)$ & $(20.7 \%)$ \\
Lost to follow-up & 4 & 4 \\
Death due to TB & 0 & 1 \\
MDR-TB & 0 & 1 \\
Others & 8 & 14 \\
Death not related to TB & 1 & 3 \\
Transferred & 7 & 11
\end{tabular}

SSM: sputum smear microscopy; TB: tuberculosis; MDR: multi drug resistance.

TABLE 4: - Average cost of sputum smear microscopy and Xpert (values in US $\$ 1=2.32$ Reals, 2012) according to the different categories.

\begin{tabular}{lcc}
\hline Cost item & SSM & Xpert \\
\hline Equipment & 0.08 & 6.81 \\
Human resources & 4.61 & 2.06 \\
Supplies & 2.24 & 15.34 \\
Infrastructure & 0.05 & 0.39 \\
\hline Estimated average cost & 6.98 & 24.61 \\
\hline
\end{tabular}

SSM: sputum smear microscopy

This was also associated with a higher mean cost (US\$24.61). In contrast, patients who were analyzed using SSM (stage 2), experienced an average of 8 days from time of triage to the initiation of TB treatment, and this was associated with a lower mean cost (US\$6.98). These results are different from those described in the literature ${ }^{5,712}$. This discrepancy is probably related to the fact that the primary data here were collected routinely and not during a clinical trial. The health unit where the data were collected participated in a previous pragmatic trial conducted in Rio de Janeiro and Manaus. Using secondary data collected in the Integrated Laboratory System and Disease Notification system, they found that the time elapsed between triage and the beginning of TB treatment was lower for patients assessed using Xpert ( 8 days) compared to the SSM (11 days) ${ }^{12}$. In this study, the Xpert and SSM tests were performed at Policlínica Augusto Amaral Peixoto, but not mycobacterial culture. When we conducted the current study, only patients with culture results were included in the analysis. Delays were observed for patients assessed using Xpert results, since the equipment was moved to another health unit, whereas the SSM analysis was retained at Policlínica Augusto Amaral Peixoto . These results reinforce the argument that, in any analysis of the incorporation of new diagnostic technologies, it is fundamental to take into account all of the care process indicators related to the diagnostic and treatment cascade, in order to analyze the patients and clinical sample flow-through of locally collected primary data, as has been reported by other authors ${ }^{5,-9}$. 
In our study, since we used only the mean cost, and not the activity based cost $(\mathrm{ABC})$, we adopted the apportionment method to cope with any distortions, when we consider the proportion of the laboratory expenses with the exam considered. We observed the cost with Xpert (US\$24.61) to be higher than the cost reported by Pinto et al.(US $\$ 17.35)^{13}$ in our country, and by Shah et al. ${ }^{23}$ (\$14.93), but lower than the cost (US\$60-61) reported in South Africa $^{24}$. However, since it was not possible to perform a costeffectiveness analysis, we cannot compare our data with the unfavorable data reported by Vassal et al. ${ }^{16}$ and by Pinto et al ${ }^{25}$.

In stages 1 and 2, the sensitivities of Xpert and SSM were $91.7 \%(22 / 24)$ and $79.1 \%(34 / 43)$.

(34/43), respectively, similar to those described in a recent meta-analysis, which analyzed studies comparing the two technologies, where the sensitivity of Xpert $(88 \%)$ was higher than that of SSM $(70.0 \%)^{26}$.

The proportion of TB (29.7\%) identified in our study among the probable pulmonary TB cases is similar to that which has been described previously in the same health unit ${ }^{27}$. The proportion of empirical treatments was higher with Xpert (7.7\%), but was lower than that described in other series ${ }^{5,12}$.

The adoption of the Xpert molecular test, with a higher diagnostic yield, did not provide more favorable TB treatment outcomes, as has been described in other studies ${ }^{5,7,14}$. The proportion of patients lost to follow-up (11.9\%) was high, but similar to that described in the City of Rio de Janeiro ${ }^{16}$.

In the present study, the majority of the patients were young male adults, of low socioeconomic status with low education levels, similar to the patient population described previously ${ }^{1,16,28,29}$.In our pragmatic, prospective study, primary data were collected either through interviewing subjects with probable pulmonary tuberculosis, as well as through secondary laboratory data and the TB treatment outcomes, available from the laboratory electronic system and the Disease Notification System, respectively.

The limitations of this study include the following: a) a small number of subjects with probable pulmonary tuberculosis attended only one health unit in the City of Rio de Janeiro; b) no data was collected on patient costs related to TB diagnosis; these costs should be evaluated in the future, because of significant and growing socioeconomic inequality, the free diagnosis and treatment of TB is not sufficient to alleviate the financial limitations to which most patients are exposed. For many patients, expenditures related to TB care (transportation, food, etc.) can be catastrophic, so this should be evaluated in future studies; and c) we did not perform a qualitative evaluation to analyze the barriers or facilitators factors to incorporating the use of the Xpert molecular test compared to smear microscopy.

In addition, our results call attention to the importance of rapid tests such as Xpert, when routinely incorporated. A shorter time is required to release the data for the detection of the Mycobacterium tuberculosis complex. The test also allows for the detection of rifampicin resistance (a molecular marker of MDR-TB). The SSM test only detects the presence or absence of mycobacteria, and cannot differentiate non-tuberculous
Mycobacteria from the Mycobacterium tuberculosis complex. However, failures in the operation of health services, such as those observed in our study, may negatively affect the potential utilization of new technologies such as Xpert. With a new promising technology that has been recommended by WHO, and before its incorporation into the health system, the results of our study highlight the need to evaluate the clinical impacts and the costs of any new technology in association with care processes adopted by the local health system, under field conditions ${ }^{7-10}$.

In conclusion, in high-burden countries, it is necessary to identify, through primary data, at both the regional and local level, the most effective and cost-effective diagnostic strategies that can expedite the TB diagnosis and the initiation of appropriate TB treatment, thereby lowering the transmission of TB in the community and promote the sustainability of the procedures adopted.

\section{Conflict of interest}

The authors declare that there is no conflict of interest.

\section{Financial support}

This work was funded by Conselho Nacional Pesquisa (CNPq)/Institutos Nacionais de Ciência e Tecnologia (INCT) - Process: 465318/2014-2.

\section{REFERENCES}

1. World Health Organization (WHO). Global tuberculosis report 2016. Geneva: WHO; 2016.

2. World Health Organization (WHO). Integrating collaborative TB and HIV services within a comprehensive package of care for people who inject drugs: consolidated guidelines. WHO/HTM/TB/2016.02. http://apps.who.int/iris/bitstream/10665/204484/1/9789241510226_ eng.pdf?ua $=1$

3. World Health Organization (WHO). Use of liquid TB culture and drug susceptibility testing (DST) in low and medium income settings. Summary Report of the expert group meeting on the use of liquid culture media. Geneva: World Health Organization, 2007.

4. World Health Organization (WHO). Moving research findings into new WHO policies reference: World Health Organization. Moving research findings into new WHO policies. http://www.who.int/tb/ dots/laboratory/policy/en/index4.html. 2008.

5. Boyles TH. Why do clinical trials of Xpert ${ }^{\circledR}$ MTB/RIF fail to show an effect on patient relevant outcomes? Int J Tuberc Lung Dis. 2017;21(3):249-50.

6. Vassall A, Siapka M, Foster N, Cunnama L, Ramma L, Fielding $\mathrm{K}$, et al. Cost-effectiveness of Xpert MTB/RIF for tuberculosis diagnosis in South Africa: a real-world cost analysis and economic evaluation. Lancet Glob Health. 2017;5(7):e710-e719.

7. Churchyard GJ, Stevens WS, Mametja LD, McCarthy KM, Chihota $\mathrm{V}$, Nicol MP, et al. Xpert MTB/RIF versus sputum microscopy as the initial diagnostic test for tuberculosis: a cluster randomised trial embedded in South African roll-out of Xpert MTB/RIF. Lancet Glob Health. 2015;3(8):450-7.

8. Langley, I, Squire SB, Dacombe R, Madan J, Lapa e Silva, JR, Barreira D, et al. Developments in impact assessment of new diagnostic algorithms for tuberculosis control. Clin Infect Dis. 2015;61(Suppl 3):S126-34. 
9. Albert H, Nathavitharana RR, Isaacs $\mathrm{C}$, Pai $\mathrm{M}$, Denkinger $\mathrm{CM}$, Boehme CC. Development, roll-out and impact of Xpert MTB/RIF for tuberculosis: what lessons have we learnt and how can we do better? Eur Respir J. 2016;48(2):516-25.

10. Scatena LM, Villa TCS, Ruffino Netto A, Kritski AL, Figueiredo RMRM, Vendramini SHF, et al. Dificuldades de acesso a serviços de saúde para diagnóstico de tuberculose em municípios do Brasil. Rev Saúde Pública. 2009;43(3):389-97.

11. Ministério da Saúde (MS). Secretaria de Vigilância em Saúde. Indicadores prioritários para o monitoramento do Plano Nacional pelo Fim da Tuberculose como Problema de Saúde Pública no Brasil. Boletim Epidemiológico.2017;48(8):1-11.http://portalarquivos. saude.gov.br/images/pdf $/ 2017 / \mathrm{marco} / 23 / 2017-\mathrm{V}-48-\mathrm{N}-8$ Indicadoresprioritrios-para-o-monitoramento-do-Plano-Nacionalpelo-Fim-da-Tuberculose-como- Problema-de-Saude-Publica-noBrasil.pdf.

12. Durovni B, Saraceni V, van denHof S, Trajman A, Cordeiro-Santos $\mathrm{M}$, Cavalcante S, et al. Impact of replacing smear microscopy with Xpert MTB/RIF for diagnosing tuberculosis in Brazil: a steppedwedge cluster-randomized trial. PLoS Med. 2014; 9:11(12):e1001766.

13. Pinto M. Entringer AP, Steffen R, Trajman A. Cost analysis of nucleic acid amplification for diagnosing pulmonary tuberculosis, within the context of the Brazilian Unified Health Care System. J Bras Pneumol. 2015;41(6):536-8.

14. Trajman A, Durovni B, Saraceni V, Menzies A, Cobelens F, van den Hof S. Impact on patient's treatment outcomes of Xpert MTB/ RIF implementation for the diagnosis of tuberculosis: followup of a stepped-wedge randomized clinical trial. Plos one. 2015; 27:10(4):e0123252.

15. Ministério da Saúde (MS). Biblioteca Virtual em Saúde. Portaria MS no 48 de 10 de setembro de 2013. Brasília:MS; 2013. Citado:3 dez 2014. Disponível em:http://www.bvsms.saude.gov.br/bvs/ saudelegis/sctie/2013/prt0048_10_09_2013.html.

16. Ministério da Saúde (MS). Secretaria de Vigilância em Saúde. Departamento de Vigilância Epidemiológica. Manual de recomendações para o controle da tuberculose no Brasil. Brasília:MS;2011.288p.

17. Kent PT, Kubica GP. Public Health Mycobacteriology: aGuide for the Level III Laboratory. National Technical Reports Library. U.S. Department of Commerce. Washington, DC: 1985. 226p.

18. Cepheid Brasil. A better way. The New GeneXpert ${ }^{\circledR}$ System. New Systems. Same game-changing performance. http://www.cepheid. com/administrator/components/com_productcatalog/libraryfiles/9a.

19. Ministério da Saúde (MS). Secretaria de Vigilância em Saúde. Diretrizes Metodológicas: estudos de avaliação econômica de tecnologias em saúde Ministério da Saúde, Secretaria de Ciência, Tecnologia e Insumos Estratégicos, Departamento de Ciência e Tecnologia. Brasília: MS;2009. 145p.

20. Alinski ML, Young HP. A new method for congressional apportionment. Proc Natl Acad Sci USA.1974;71(11):4602-6.

21. Ministério da Saúde (MS). Secretaria de Vigilância em Saúde. Departamento de Vigilância Epidemiológica. Manual Nacional de Vigilância Laboratorial da Tuberculose e outras Micobactérias. Brasília:MS; 2008. 436p. Disponível em: http://portal.saude.gov.br/ portal/arquivo/pdf/manual_laboratorio_tb_8_05_13.pdf.

22. Ministério da Fazenda (MF). Banco Central do Brasil. Cotações e boletins. Brasília:MF; 2018. Acesso: 26 fev 2018.http://www4.bcb. gov.br/pec/taxas /port/ptaxnpesq.asp.

23. Shah M, Chihota V, Coetzee G, Churchyard G, Dorman SE. Comparison of laboratory costs of rapid molecular tests and conventional diagnostics for detection of tuberculosis and drug resistant tuberculosis in South Africa. BMC Infect Dis. 2013;13:352.

24. Meyer-Rath G, Schnippel K, Long L, W, Sanne I, Stevens W, Pillay $\mathrm{S}$, et al. The impact and cost of scaling up Gene Xpert MTB/RIF in South Africa. PLoS One. 2012;7(5):e36966.

25. Pinto M, Steffen RE, Cobelens F, van den Hof S, EntringerA, Trajman A. Cost effectiveness of the Xpert ${ }^{\circledR}$ MTB/RIF assay for tuberculosis diagnosis in Brazil. Int J TubercLung Dis.2016;20(5):611-8.

26. Steingart KR, Schiller I, Horne DJ, Pai M, Boehme CC, Dendukuri N. Xpert ${ }^{\circledR}$ MTB/RIF assay for pulmonary tuberculosis and rifampicin resistance in adults. Cochrane Database Syst Rev. 2014;21;(1):CD009593.

27. Castro CB, Costa PA, Ruffino-Netto A, Maciel EL, Kritski AL. Assessment of a clinical score for screening suspected pulmonary tuberculosis cases. Rev Saúde Pública. 2011;45(6):1110-6.

28. Lönnroth K, Castro KG, Chakaya JM, Chauhan LS, Floyd K, Glaziou P, et al. Tuberculosis control and elimination 2010-50: cure, care, and social development. Lancet. 2010;375(9728):1814-29.

29. Yamamura M, Santos Neto M, Freitas IM, Rodrigues LBB, Popolin MP, Uchoa SAC, et al. Tuberculose e iniquidade social em saúde: uma análise ecológica utilizando técnicas estatísticas multivariadas, São Paulo, Brasil. Rev Panam Salud Publica. 2014;35(4):2707. 